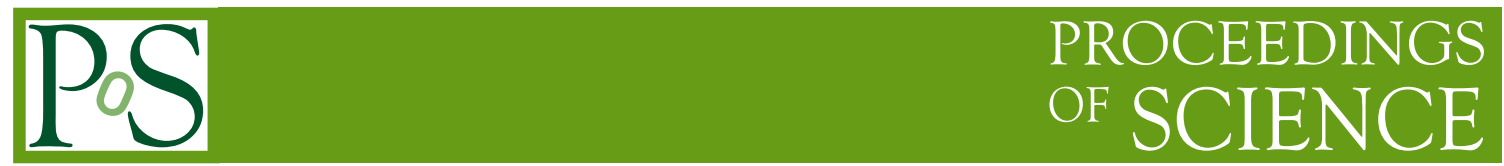

\title{
Search for new phenomena in top quark interactions
}

\section{K. Skovpen (on behalf of the ATLAS and CMS Collaborations) ${ }^{a}$}

${ }^{a}$ Ghent University,

Proeftuinstraat 86, B-9000 Gent, Belgium

The study of the processes with the production of top quarks represents a unique possibility to test the standard model (SM) predictions and probe the new physics effects. Potential deviations from the SM expectations are parametrized within the framework of the Effective Field Theory (EFT). The latest EFT results from ATLAS and CMS experiments are presented. Dedicated studies of processes with flavour-changing neutral currents are also discussed.

The Eighth Annual Conference on Large Hadron Collider Physics-LHCP2020

25-30 May, 2020

online 
The top quark sector plays an important role in precision tests of the standard model (SM) predictions and searches for new physics. The heaviness of the top quark and its large coupling strength of the interaction with the Higgs boson suggests an enhanced sensitivity to hypothetical new heavy particles and interactions. The potential new physics effects can be probed in the study of the SM processes with top quarks, as well as by searching for forbidden interactions of these elementary particles. This report summarizes the latest experimental results on these studies from the ATLAS [1] and CMS [2] experiments using proton-proton collision data collected at $\sqrt{s}=13 \mathrm{TeV}$ at the LHC.

The Effective Field Theory (EFT) approach represents a generalized parametrization of various new physics effects on the basis of dimension-six operators [3, 4]. The SMEFT framework provides a description of all possible interaction vertices involving the SM elementary particles [5]. The processes involving the so-called flavour-changing neutral currents (FCNC) are among possible anomalous interactions of top quarks, which are also included in the EFT description [6]. These interactions are forbidden in the SM at tree level and are significantly suppressed in higher orders [7].

Processes with the production of top quark pairs (tit), as well as the associated production of a top quark with a W boson (tW), are sensitive to several EFT operators involving the top quark. The first simultaneous study of these two production modes is performed in dilepton opposite-sign events using $36 \mathrm{fb}^{-1}$ of the data collected by CMS [8]. The analysis probes the anomalous Wtb and FCNC couplings in the tW production mode, while the triple gluon field interactions are studied in the $\bar{t}$ production channel. Additionally, the chromomagnetic dipole moments of the top quark are probed in both production modes. The study of EFT effects is optimized using neural network (NN) discriminants. The NN distributions are used to extract the constraints on the relevant EFT Wilson coefficients through a likelihood fit to data of the expected signal and background contributions (Fig. 1).

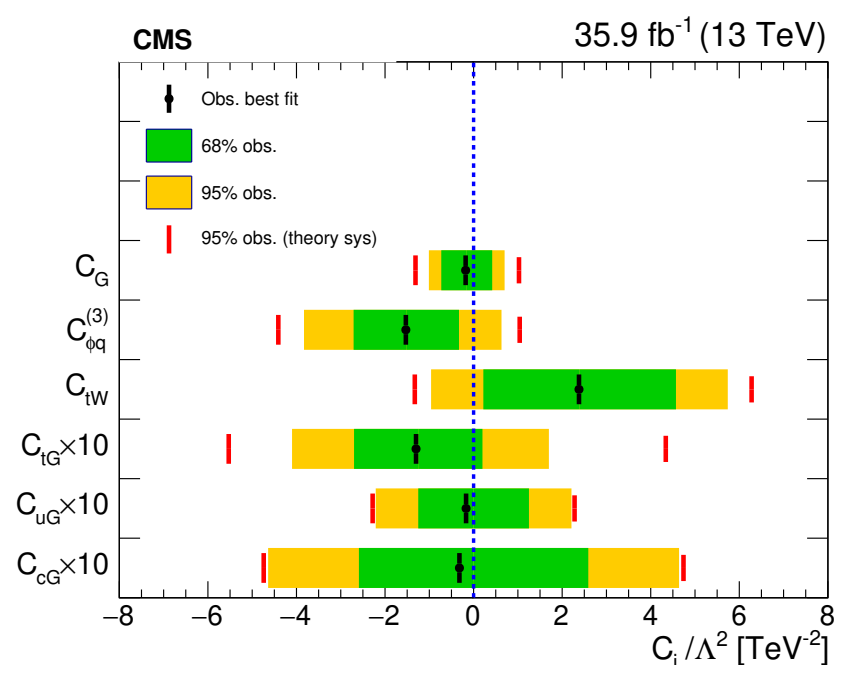

Figure 1: The observed constraints on the EFT Wilson coefficients in the analysis [8]. The dashed line shows the SM expectation value. The theory uncertainty here encodes the uncertainties on the theoretical predictions for the signal cross section arising from the choice of factorization and renormalization scales as well as parton distribution functions. One non-vanishing Wilson coefficient is assumed at a time. 
The chromomagnetic and chromoelectric dipole moments of the top quark are studied in the opposite-sign dilepton analysis of the $\bar{t} \bar{t}$ spin correlations and differential production cross sections with $36 \mathrm{fb}^{-1}$ of CMS data [9]. The full spin density matrix is measured to probe various anomalous effects of the four-particle effective couplings with top quarks. The resulting constraints on the chromomagnetic and chromoelectric moments are $-0.24<\mathrm{C}_{\mathrm{tG}} / \Lambda^{2}<0.07 \mathrm{TeV}^{-2}$ and $-0.33<$ $\mathrm{C}_{\mathrm{tG}}^{\mathrm{I}} / \Lambda^{2}<0.20 \mathrm{TeV}^{-2}$, respectively. The obtained results represent a significant improvement over the previously published direct limits.

A model independent search for top quark charged lepton flavour violation (cLFV) decays is performed in the final states with three leptons using $80 \mathrm{fb}^{-1}$ of the ATLAS data [10]. The analysis is sensitive to the axial-vector, scalar, pseudo-scalar, and lepton-quark EFT operators. A Boosted Decision Tree (BDT) discriminant is used to suppress the SM backgrounds. The observed (expected) resulting constraints on the top quark cLFV branching fraction, $\mathrm{B}\left(\mathrm{t} \rightarrow 1 \mathrm{l}^{\prime} \mathrm{q}\right)<1.9(1.4) \cdot 10^{-5}$, improve over the best indirect limits by several orders of magnitude.

The recent studies of the top quark FCNC interactions with a photon $(\gamma)$ using $81 \mathrm{fb}^{-1}$ of the ATLAS data have led to significantly improved constraints on these types of interaction [11]. The analysis is performed in the single lepton final states with a photon. The FCNC effects are simultaneously probed in the top quark decays in $\mathrm{tt}$ events, as well as in the associated production of the top quark with a photon via anomalous FCNC couplings. The NN discriminant is used to suppress the $\mathrm{W} / \mathrm{Z}+\gamma$ processes, as well as events with mis-identified photons. The observed (expected) limits on the top quark FCNC decay branching fraction for left- and right-handed couplings with an up-quark are $\mathrm{B}(\mathrm{t} \rightarrow \mathrm{u} \gamma)<2.8(4.0) \cdot 10^{-5}$ and $\mathrm{B}(\mathrm{t} \rightarrow \mathrm{u} \gamma)<6.1(5.9) \cdot 10^{-5}$, respectively. In the case of the top quark FCNC couplings with a charm quark, the corresponding limits are $\mathrm{B}(\mathrm{t} \rightarrow \mathrm{c} \gamma)<22(27) \cdot 10^{-5} \mathrm{~B}(\mathrm{t} \rightarrow \mathrm{c} \gamma)<16(28) \cdot 10^{-5}$, respectively.

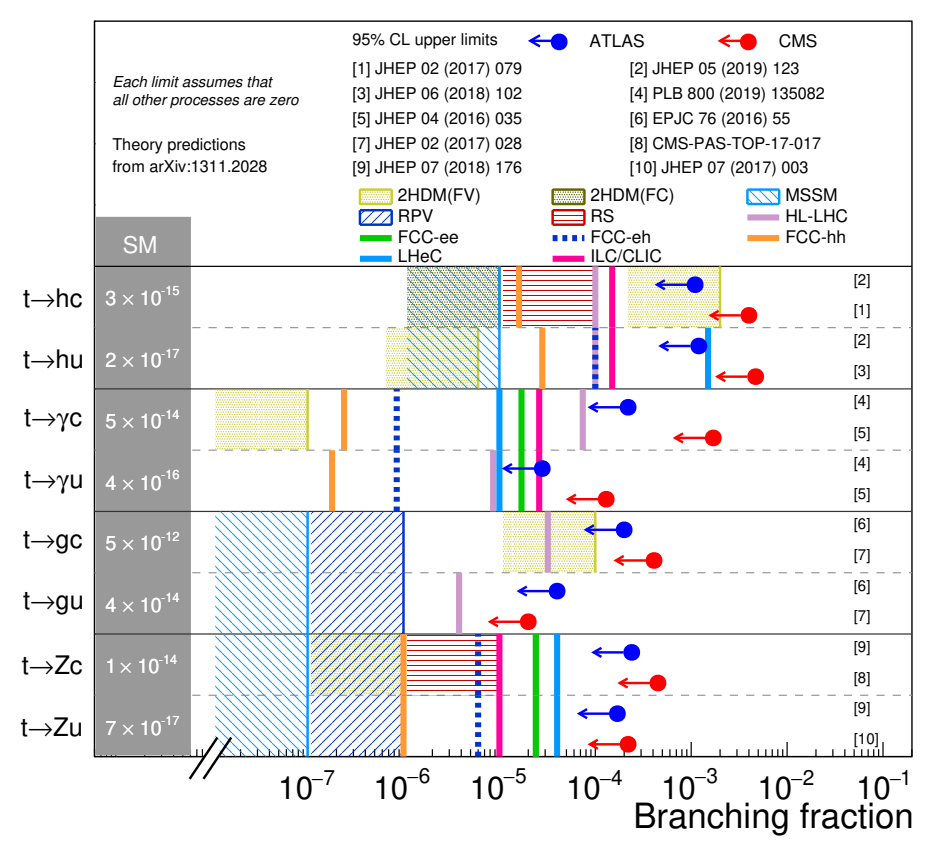

Figure 2: The updated summary of the FCNC searches with top quarks from Ref. [13]. 
The combination of the ATLAS results obtained from the studies of various final states relevant to the top quark FCNC interactions with the Higgs boson (h) is performed [12] with $36 \mathrm{fb}^{-1}$ of recorded data. The considered final states arise from the Higgs boson decays to two b quarks, two tau leptons, WW and ZZ boson pairs, and two photons. The obtained observed (expected) limits on the top FCNC decays via a Higgs boson with an up and a charm quark are $\mathrm{B}(\mathrm{t} \rightarrow \mathrm{uh})<1.2(0.8) \cdot 10^{-3}$ and $\mathrm{B}(\mathrm{t} \rightarrow \mathrm{ch})<1.1(0.8) \cdot 10^{-3}$, respectively. These results represent the best limits to date on top-Higgs FCNC interactions.

The summary of the latest constraints obtained with the LHC data on the top quark FCNC branching ratios, along with the theoretical predictions [6] and projections of sensitivity of experimental searches for future experiments [13-15], is presented in Fig. 2. The shown experimental limits represent an important probe of various models of new physics.

The presented experimental results on the EFT and FCNC searches with top quarks show a great improvement over the previously published results, as well as introduce novel approaches of studying the top quark anomalous interactions. The described results were obtained using the partial data collected during the second run of the data taking at the LHC, and further updates from the analysis of the full data are foreseen in the future.

\section{References}

[1] ATLAS Collaboration, JINST 3 (2008) S08003.

[2] CMS Collaboration, JINST 3 (2008) S08004.

[3] W. Buchmuller and D. Wyler, Nucl. Phys. B 268 (1986) 621.

[4] B. Grzadkowski, M. Iskrzynski, M. Misiak et al., JHEP 10 (2010) 085.

[5] J. A. Aguilar-Saavedra et al., arXiv:1802.07237.

[6] J. A. Aguilar-Saavedra, Acta Phys. Polon. B 35 (2004) 2695.

[7] S. L. Glashow, J. Iliopoulos, and L. Maiani, Phys. Rev. D 2 (1970) 1285.

[8] CMS Collaboration, Eur. Phys. J. C 79 (2019) 886.

[9] CMS Collaboration, Phys. Rev. D 100 (2019) 072002.

[10] ATLAS Collaboration, ATLAS-CONF-2018-044, http://cds.cern.ch/record/2638305.

[11] ATLAS Collaboration, Phys. Lett. B 800 (2019) 135082.

[12] ATLAS Collaboration, JHEP 05 (2019) 123.

[13] A. Abada et al., Eur. Phys. J. C 79 (2019) 474.

[14] P. Azzi et al., arXiv:1902.04070.

[15] A. Cerri et al., arXiv:1812.07638. 